\title{
Cyclostationary Analysis of ECG Signals Acquired Inside an Ultra-High Field MRI Scanner
}

\author{
Michel Haritopoulos \\ PRISME Laboratory, EA 4229 \\ 21, rue de Loigny la Bataille \\ 28000 Chartres, France \\ Email: michel.haritopoulos@univ-orleans.fr
}

\author{
Johannes Krug \\ and Alfredo Illanes \\ and Michael Friebe \\ INKA Intelligente Katheter \\ Otto von Guericke University \\ Magdeburg, Germany
}

\author{
Asoke K. Nandi \\ Department of Electronic and \\ Computer Engineering \\ Brunel University London \\ Uxbridge, UK
}

\begin{abstract}
In this paper, a strategy is proposed to estimate the R-peaks in ECG signals recorded inside a $7 \mathrm{~T}$ magnetic resonance imaging (MRI) scanner in order to reduce the disturbances due to the magnetohydrodynamic (MHD) effect and to finally obtain high quality cardiovascular magnetic resonance (CMR) images. We first show that the cyclostationarity property of the ECG signal disturbed by the MHD effect can be quantified by means of cyclic spectral analysis. Then, this information is forwarded as input to a cyclostationary source extraction algorithm applied to a set of ECG recordings acquired inside the MRI scanner in a Feet first (Ff) and a Head first (Hf) positions. Finally, detection of the R-peaks in the estimated cyclostationary signal completes the proposed procedure. Validation of the method is performed by comparing the estimated with clinical R-peaks annotations provided with the real world dataset. The obtained results are promising and future research directions are discussed.
\end{abstract}

\section{INTRODUCTION}

Introduced in the early 1980s, Magnetic Resonance Imaging (MRI) is nowadays widely used in clinical practice and Cardiovascular Magnetic Resonance (CMR) is a non invasive medical imaging technique regarded as a powerful diagnostic tool in cardiology that aims to create still or animated (Cine Imaging) imaging sequences of the heart during the cardiac cycle. Synchronization of data acquisition with the cardiac cycle is necessary for imaging the heart because it is a constantly moving organ. Without any synchronization, the resulting Magnetic Resonance (MR) image would be strongly affected by motion blur. For synchronization purposes, measurements of the cardiac activity through electrocardiograms (ECGs) are made simultaneously with MRI. The final MR image is formed by segments acquired over consecutive R-R intervals.

CMR studies were intially performed at a field strength of 1.5 Tesla until the United States Food and Drug Administration (US FDA) approved in 2002 using 3 Tesla (3 T) MRI scanners for whole body imaging [1]. Nowadays, $3 \mathrm{~T}$ MRI scanners are well established in clinical routine, but $7 \mathrm{~T}$ MRI scanners are also used mainly for research.

The main challenges to performing $\mathrm{CMR}$ at $7 \mathrm{~T}$ are the various artifacts present in the image. These are due to the heart's movement, the respiratory cycle, and the prolonged scanning times. In addition to that, the high field magnetic strength introduces a phenomenon called the magnetohydrodynamic
(MHD) effect distording the electrocardiogram. Indeed, ECGbased gating is a crucial step for acquiring high quality CMR images as the $\mathrm{R}$ wave extraction is of great importance for a perfect synchronisation of the image with the heart motion.

Several methods are found in the dedicated literature based on the vectorcardiogram (VCG) [2] and on Blind Source Separation (BSS) [3] techniques, but to our knowledge none uses a statistical property characterising ECG signals, called cyclostationarity, to overcome the challenge of R-peak detection in presence of the MHD effect. The work presented here is a first approach to the problem of ECG-based gating in ultra high field CMR.

In previous work [4] the assumption of ECG signals being cylcostationary has been validated within the framework of extracting the fetal electrocardiogram (FECG) from a set of ECGs coming from electrodes attached on a pregnant woman. In fact, the fetus' as well as the mother's heartbeats are not stricltly periodic, but they both exhibit some hidden periodicities, i.e. their statistical characteristics vary periodically with time. In the proposed work, this property is tested to real world ECG signals recorded inside a $7 \mathrm{~T}$ MRI scanner and a cyclostationarity-based source extraction algorithm is used in order to overcome the artifacts cited above in the R-peak detection step.

In Section II, the property of cyclostationarity and one of its quantitative measures are introduced and illustrated on real world signals; moreover, a brief introduction to BSS theory and one source extraction algorithm tailored for cyclostationary signals are introduced. The proposed procedure for Rpeak detection is presented in Section III, while in Section IV experimental results from the application of the proposed procedure to a real world ECG dataset are displayed. Finally, a conclusion and a discussion of future research direction are provided in Section V.

\section{CyClostationary nature OF ECG MRI Signals}

\section{A. The property of cyclostationarity}

An introduction to cyclostationary processes can be found in [5] while Gardner et al offered important contributions to the development of the theory of cyclostationary signals [6] with a focus to telecommunications. Since then, other applications 

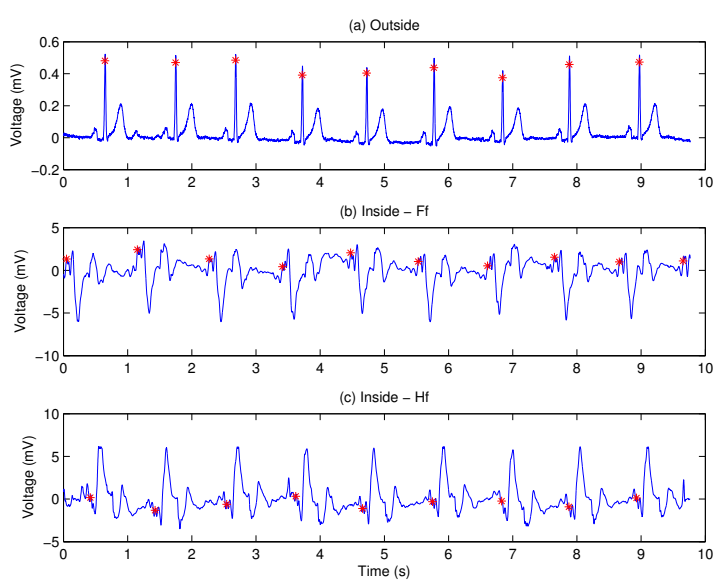

Fig. 1. Time waveforms of 3 ECGs recorded from lead $I$ for volunteer number 3 and acquired outside (a) and inside the MRI scanner in Ff (b) and in Hf (c) positions. "Star" markers indicate clinically annotated R-peaks.

of this theory have emerged in numerous and varied fields such as rotating machinery [7], econometrics [8] or even radioastronomy [9]. Reviews on cyclostationarity can be found in [10], [11], [12].

Cyclostationarity is a special case of non-stationarity: a time-variant process whose statistics vary periodically with time is called cyclostationary (CS). For example, the periodicity of the cosine function, $\cos \left(2 \pi f_{0} t\right)$, at some carrier $f_{0}$ becomes hidden if its amplitude is modulated by some random data $x(t)$ with autocorrelation function $R_{x}(\tau)=$ $E[x(t) x(t+\tau)]$.

\section{B. Cyclic coherence's definition and integrated cyclic coher-} ence

Let $x(t)$ be a CS signal. In order to characterize the cyclostationarity of $x(t)$, the measure proposed in this work is a normalized version of the spectral correlation function, called cyclic coherence (CC) $C_{x x}(f, \alpha)$ :

$$
C_{x x}(f, \alpha)=\frac{S_{x x}(f, \alpha)}{\left[S_{x x}(f-\alpha / 2) S_{x x}(f+\alpha / 2)^{*}\right]^{1 / 2}}
$$

where the $*$ operator denotes the complex conjugate, $t$ and $\tau$ stand for time index and time-lag, respectively, $f$ and $\alpha$ denote spectral and cyclic frequencies, respectively, and $S_{x x}(f, \alpha)$ is the Fourier Transform of the cyclic autocorrelation function $R_{x x}^{\alpha}(\tau)$ :

$$
S_{x x}(f, \alpha)=\int_{-\infty}^{+\infty} R_{x x}^{\alpha}(\tau) e^{-j 2 \pi f \tau} d \tau
$$

The cyclic coherence $C_{x x}(f, \alpha)$ of eq. (1) is computed using the averaged cyclic periodogram technique [13] and it simply indicates how strong the correlation between components of $x(t)$ at frequencies $(f-\alpha / 2)$ and $(f+\alpha / 2)$ is.

Another measure of cyclostationarity is the integrated Cyclic Coherence (iCC) over spectral frequency $f$ defined as:

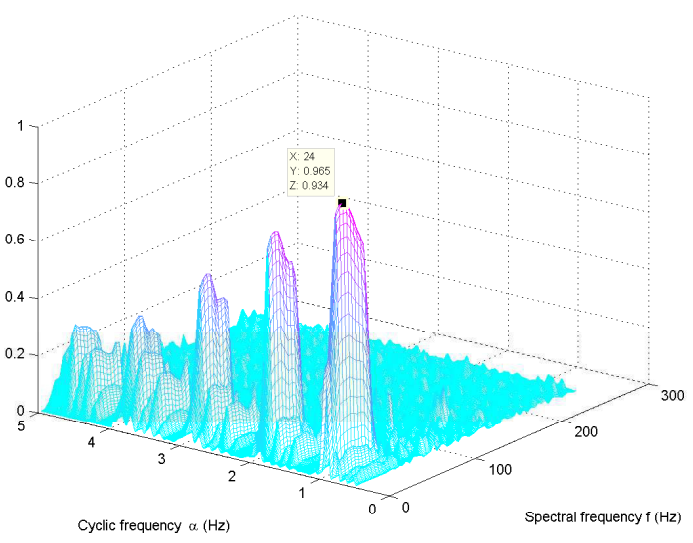

Fig. 2. Cyclic Coherence for the ECG recorded from lead $I$ of volunteer number 3 recorded outside the MRI scanner.

$$
i C C(\alpha)=\sum_{f=f_{\min }}^{f=f_{\max }} C_{x x}(f, \alpha), \alpha \in\left[\alpha_{\min }, \alpha_{\max }\right] .
$$

\section{Example data}

Let us illustrate the concept of cyclostationarity discussed above on actual data recorded using a 12-lead Holter ECG with a sampling rate of $1024 \mathrm{~Hz}$ from healthy volunteers; for a detailed description of the data acquisition procedure, readers are referred to [14]. ECGs were recorded outside but also inside the MRI scanner in Feet First (Ff) and Head first (Hf) positions that influence the way the MHD effect disrupts the signals as displayed in Fig. 1 (from top to bottom).

Plot (a) in Fig. 1 shows noise-free PQRST complexes from ECGs acquired outside the MR scanner, while the presence of MHD noise - mainly, makes it difficult for a non expert eye to locate the R-peaks as it is shown in plots (b) and (c), hence the importance of an accurate R-peak detection to trigger the MR image acquisition. The underlying physical phenomenon in these ECGs, i.e. the changing electrical potential of the heart, make of these signals CS processes as will be shown below.

The CC function of eq. (1) is computed for the ECG recorded outside the MRI scanner which is plotted in Fig. 1(a); its three-dimensional plot is illustrated by Fig. 2. For each spectral frequency value $f \in] 0,250] \mathrm{Hz}$ its correlation with every cyclic frequency $\alpha \in] 0,5] \mathrm{Hz}$ varying by a step of $5 \times 10^{-3} \mathrm{~Hz}$ is calculated. This results in the presence of a first cluster of spectral lines with high magnitude around the cyclic frequency $\alpha_{0}=0.965 \mathrm{~Hz}$ - called fundamental cyclic frequency, and of its first two harmonics one can clearly remark at approximately $\left\{2 \times \alpha_{0}, 3 \times \alpha_{0}\right\} H z$, respectively. Correlation between spectral and cyclic components tends to fade for every other value of cyclic frequency $\alpha$. This fundamental cyclic frequency reveals the quasi-periodicity of the heart beats for volunteer number 3 which is equal to $T_{0}=1 / \alpha_{0}=1.036 \mathrm{~s}$. 


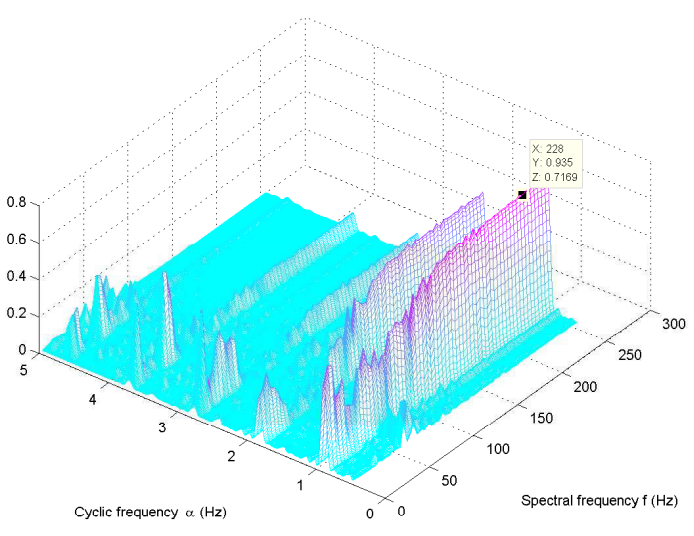

Fig. 3. Cyclic Coherence for the ECG recorded from lead $I$ of volunteer number 3 recorded inside the MRI scanner in Head first position.

Despite the MHD effect, the ECG signals recorded inside the $7 \mathrm{~T}$ MRI scanner still exhibit the cyclostationarity property. Fig. 3 shows the $\mathrm{CC}$ of the same lead and for the same volunteer as previously but for the ECG signal recorded inside the MRI scanner in Hf position. The presence of a strong correlation between all spectral frequencies and the cyclic frequency at around $0.935 \mathrm{~Hz}$ reveals a fundamental cyclic frequency $\alpha_{0}$. Its four first harmonics are also clearly visible in this plot. Obtained results are quite similar for the $\mathrm{CC}$ of the corresponding ECG recorded in Ff position.

In order to quantify more simply and accurately the fundamental cyclic frequency $\alpha_{0}$ we computed the iCCs of the three ECGs coming from lead 1 of volunteer number 3 according eq. (3). Fig. 4 displays the obtained results. For the reference lead (i.e. recorded outside the scanner) the obtained fundamental cyclic frequency plotted in solid line has a value of $0.965 \mathrm{~Hz}$, while the obtained $\alpha_{0}$ values for the ECGs recorded inside the scanner in Ff (dotted line) and Hf (dashed-dotted line) positions are $\{0.925,0.935\} \mathrm{Hz}$, respectively. Amplitudes of the computed iCCs depend strongly on the spectral frequencies range $f_{\text {max }}$.

\section{BSS and cyclostationarity-based signal extraction}

BSS [15], [16] and its underlying mathematical tool called Independent Component Analysis (ICA) [17] are well known techniques and a wide variety of strategies are based on these concepts in order to extract a signal of interest (SoI) from a set of $M$ recordings called mixtures to which contribute $N$ unknown sources $s_{i}(t), i \in[1, N]$, with $N \leq M$, and whose SoI is part. This problem is commonly modeled by the linear instantaneous noiseless BSS model which writes in matrix form:

$$
\mathbf{x}(t)=\mathbf{A s}(t)
$$

where $\mathbf{s}(t)=\left[s_{1}(t), s_{2}(t), \ldots, s_{N}(t)\right]^{\mathrm{T}}$ and $\mathbf{x}(t)=$ $\left[x_{1}(t), x_{2}(t), \ldots, x_{M}(t)\right]^{\mathrm{T}}$ denote the mixture and the source vectors, respectively, ${ }^{\mathrm{T}}$ and $t$ stand for the transpose operator and time, respectively, and where $\mathbf{A}$ is an unknown $M \times N$

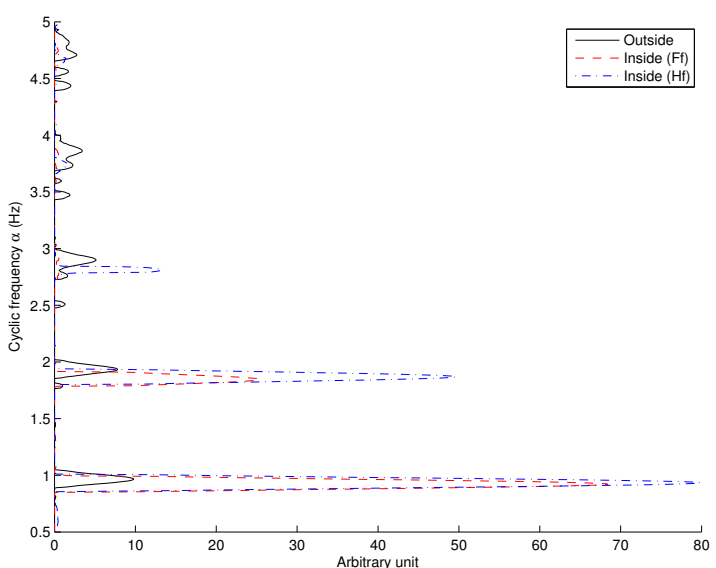

Fig. 4. Integrated Cyclic Coherence for the three ECGs from lead $I$ of volunteer number 3 recorded outside and inside the MRI scanner in Ff and Hf positions.

full rank mixing matrix. The basic assumption to solve this problem is the statistical independence of the source vector components $s_{i}$; to do so, let $\mathbf{B}$ be the estimated unmixing matrix, i.e. $\mathbf{B} \approx \mathbf{A}^{-1}$. Then, an estimate of the independent components of the vector $\mathbf{s}$ is yielded by:

$$
\hat{\mathbf{s}}(t)=\mathbf{B} \mathbf{x}(t)
$$

Within the framework of this research we attempt to exploit the cyclostationarity property of the ECG signals recorded inside the $7 \mathrm{~T}$ MRI scanner. The a priori knowledge of the fundamental cyclic frequency $\alpha_{0}$ of the SoI, i.e. the MHDfree ECG, will serve as an entry to the cyclostationary source extraction algorithm labeled as Second Order Cyclostationary Statistics Optimization Criterion $\left(\mathrm{SOC}^{2}\right.$ ) [18] which is based on the joint diagonalisation of a set of cyclic correlation matrices. This algorithm do not use whitening [19] as a pre-processing step consisting in a linear transformation of the observations vector $\mathbf{x}$ and assumes all sources can be either stationary or cyclostationary but at different fundamental cyclic frequencies.

\section{PRoposed PRocedure For R-PEAK DETECTION}

In order to estimated the R-peaks of the ECGs distorted by the MHD effect, the following procedure is proposed:

1) Estimate the fundamental cyclic frequency $\alpha_{0}$ of the ECGs recorded inside the MRI scanner both for Ff and Hf positions.

2) For each recording position ( $\mathrm{Ff}$ and $\mathrm{Hf}$ ) form a mixture vector for source extraction purposes.

3) Apply the cycloststionarity-based extraction algorithm in order to estimate the SoI, i.e. the MHD-free ECG signal.

4) Locate an R-peak.

5) Use the $\alpha_{0}$ value knowledge to estimate the rest of the R-peaks in the extracted ECG signal.

In the first step, computation of the iCC measure is performed. In the second step, and in order to reduce the 
computational cost compared to other ICA techniques, the mixture vector consists of only a part of the 12 available ECG recordings; experiments have been carried out with four leads recordings and the obtained results are provided in the next Section. These mixtures together with the $\alpha_{0}$ value are given as input to the $\mathrm{SOC}^{2}$ algorithm in step 3, yielding thus a single ECG signal supposed to be less distorted by the MHD effect. For the next step, in some experiments, all or some of the Rpeaks in the estimated SoI are easy to locate by an experienced eye. In Section IV, clinical annotations have been used in order to detect the first R-peak.

Finally, an algorithm for research of local maxima in the more or less immediate vicinity of the next R-peaks is run. As the estimated SoI is cyclostationary, the R-peaks appear after not strictly equal time intervals. Based on the $\alpha_{0}$ frequency value being estimated in step 1 , it is easy to compute the corresponding cyclic period $T_{0}$ (see Section II-C) and thus, to locate the time instant $t_{0}$ where the next R-peak should appear in the estimated SoI. A peak detection algorithm is then run on the interval $\left[t_{0}+\delta, t_{0}-\delta\right]$ and seeks for a maximum value corresponding to the next R-peak, where $\delta$ is a wiselychosen variable (in seconds) depending on the quality of the estimated ECG signal.

\section{EXPERIMENTS AND RESULTS}

As outlined above, the proposed R-peak detection procedure uses as prior knowledge the fundamental cyclic frequency $\alpha_{0}$ of the SoI. Table I summarises the results obtained for the ECGs of each volunteer recorded outside but also inside the MRI scanner in $\mathrm{Ff}$ and $\mathrm{Hf}$ positions using the procedure described in II-C. The choice of the ECG lead for iCC computation is arbitrary, as the results are essentially the same for all $12 \mathrm{ECG}$ channels. One could also compute a mean iCC value from the individual ones obtained from each ECG lead at the expense of a slightly higher computational cost.

TABLE I

ESTIMATED ICCS VALUES (IN $\mathrm{Hz}$ ) OF EACH VOLUNTEER'S ECG RECORDED OUTSIDE AND INSIDE THE MRI SCANNER IN FF AND HF POSITIONS.

\begin{tabular}{|c||c|c|c|c|c|}
\hline Volunteer & 1 & 2 & 3 & 4 & 5 \\
\hline \hline Outside & 0.825 & 1.185 & 0.965 & 1.155 & 1.145 \\
\hline Inside - Ff & 0.74 & 1.1 & 0.92 & 1.105 & 1.105 \\
\hline Inside - Hf & 0.76 & 0.995 & 0.935 & 1.11 & 1.2 \\
\hline
\end{tabular}

For the input to the cyclostationarity-based source extraction algorithm (step 2 of the proposed procedure), two mixture vectors are used for the ECG leads recordings: the first one is formed by ECG channel numbers $\{a V L, a V F, V 1, V 2\}$ for recordings inside the MRI scanner in Ff position and the second one consists of ECG channel numbers $\{I I I, a V F, V 3, V 4\}$ for recordings inside the MRI scanner in Hf position. Note that the length of the available experimental dataset varies according to the subject; recordings were made inside the MRI scanner for each position for $3.5-6$
TABLE II

THE MEAN ERROR (IN seconds) BETWEEN ESTIMATED AND CLINICALLY ANNOTATED R-PEAKS FROM THE EXTRACTED ECG FOR EACH VOLUNTEER AND THE ASSOCIATED STANDARD DEVIATION VALUES (STD) FROM RECORDINGS IN FF AND HF POSITIONS INSIDE THE MRI SCANNER.

\begin{tabular}{|c|c||c|c|c|c|c|}
\hline \multicolumn{2}{|c|}{ Volunteer } & 1 & 2 & 3 & 4 & 5 \\
\hline \hline \multirow{2}{*}{ Ff } & Error & -0.382 & 2.521 & 0.058 & $-1 \times 10^{-3}$ & -0.456 \\
& STD & 0.564 & 2.568 & 0.445 & 0.092 & 0.342 \\
\hline \hline \multirow{2}{*}{ Hf } & Error & $4 \times 10^{-6}$ & -0.273 & $7 \times 10^{-4}$ & -0.002 & 2.8 \\
& STD & $2 \times 10^{-3}$ & 0.145 & $8 \times 10^{-4}$ & $4 \times 10^{-4}$ & 1.083 \\
\hline
\end{tabular}

min. The mixture vector's choice is quite random as various experiments with different mixture vector sizes as well as components have been realised, the aim being to find a tradeoff between the computational cost and the cyclostationary SoI estimation quality of the $\mathrm{SOC}^{2}$ algorithm.

The ECG recordings inside the scanner for each volunteer are supplemented with clinical annotations of the R-peaks. In order to safely compare the results of the proposed procedure with these annotations, in step 4 of the proposed procedure the first clinically annotated R-peak is used in order to locate the first R-peak in the estimated ECG as yielded in the previous step by the cyclostationary source extraction algorithm. Next, we applied the procedure of step 5 described in the previous Section to estimate the rest of the SoI's R-peaks. Finally, the mean difference (in seconds) between the estimated time instant the R-peaks occur and the corresponding clinical annotations as well as the standard deviation values of this error are computed for each volunteer and for both positions inside the MRI scanner.

Table II summarises the obtained results. It can be noted the proposed procedure provides generally better results in the case of ECGs recorded inside the MRI scanner in Hf position (except for volunteer number 5 ). This finding may be correlated with the nature of the MHD effect which polarity changes when the body of the volunteer is placed inside the scanner in Hf position [20]. One can also remark that, in two cases (i.e. for volunteer number 2 in Ff position and volunteer number 5 in Hf position), the estimated cyclostationary signal is still correlated with the MHD effect, hence a significant mean error. In all other cases, the good quality of the extracted cyclostationary source lead to a good R-peak detection. As shown in Fig. 5, the R-peaks detected by the proposed method are in almost perfect agreement with those annotated by the experts.

Let us also note that the results displayed by Table II are obtained by applying the proposed procedure to a mixture vector of size $M=4$ among 12 available ECG electrode recordings. More experiments are needed to determine, on the one hand, the more suitable leads for high quality cyclostationarity-based extraction of the SoI and, on the other hand, the optimum number of sensors to be used as input to the aforementioned extraction algorithm. 


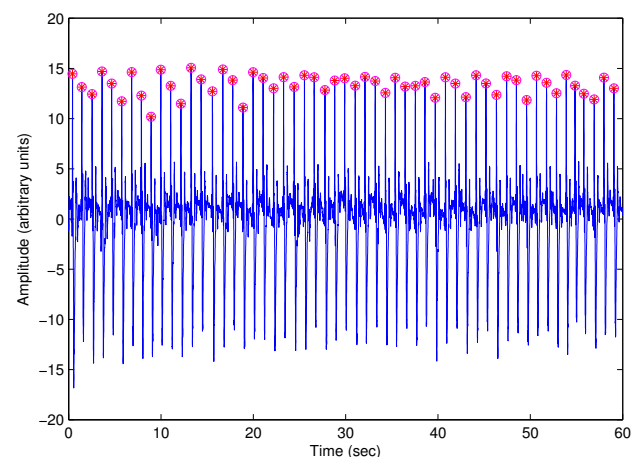

Fig. 5. Illustrative example showing the first $60 \mathrm{sec}$ of the extracted cyclostationary source using the $\mathrm{SOC}^{2}$ algorithm from the 4 channel mixture $\{I I I, a V F, V 3, V 4\}$ of volunteer number 3 recorded inside the MRI scanner in Hf position and the manually annotated R-peaks ("star" markers) vs those estimated by the proposed procedure ("circle" markers).

\section{CONCLUSION AND DISCUSSION}

Although 3 T MRI scanners are commonly used in clinical practice, research applications in CMR are using $7 \mathrm{~T}$ MRI scanners. But the image synchronisation is hampered by artifacts whose magnetohydrodynamic (MHD) effect corrupts the recorded ECGs inside the MRI scanner. The proposed work is a first step towards an automated procedure for MHD-filtered ECG signal based on the inherent cyclostationarity property of the latter.

Instead of using general blind source separation (BSS) algorithms, we propose to apply a signal extraction algorithm tailored for cyclostationary source signals within the framework of BSS. First having shown that the signal of interest (SoI), i.e., the ECG recorded inside the $7 \mathrm{~T}$ MRI scanner, still exhibits the cyclostationarity property - despite the MHD effect, the estimation of the fundamental cyclic frequency of the SoI is performed using cyclic spectral analysis tools, namely the integrated cyclic coherence (iCC).

Obtained results by application of the proposed procedure to a real world dataset of ECGs recorded outside and inside a MRI scanner in Feet first (Ff) and Head first (Hf) positions from 5 volunteers, are promising. Further studies are proposed to decide on the robustness of the method. Use of different ECG leads, of various number of electrodes to form the mixture vector, but also application of the proposed method to ECG signals distorted by real and simulated MHD effect [3] and comparison of the results with those obtained by applying well established BSS algorithms in terms of Sensitivity $(\mathrm{Se})$ and positive predictive value $(+P)$ represent some directions of future research.

\section{ACKNOWLEDGMENT}

Professor Nandi is a Distinguished Visiting Professor at The Key Laboratory of Embedded Systems and Service Computing, College of Electronic and Information Engineering, Tongji University, Shanghai, P.R. China. This work was partly supported by the National Science Foundation of China grant number 61520106006 and the National Science Foundation of Shanghai grant number 16JC1401300. This work was partly funded by the German BMBF grant 03IPT7100X.

\section{REFERENCES}

[1] E. Kanal, J. P. Borgstede, J. A. Barkovitch, C. Bell, W. G. Bradley, J. P. Felmlee, J. W. Froelich, E. M. Kaminski, E. K. Keeler, J. W. Lester, E. A. Scoumis, L. A. Zaremba, and M. D. Zinninger, "American college of radiology white paper on MR safety," American Journal of Roent, vol. 178, pp. 1335-1347, 2002.

[2] M. S. Nacif, A. Zavodni, N. Kawel, E.-Y. Choi, J. A. C. Lima, and D. A. Bluemke, "Cardiac magnetic resonance imaging and its electrocardiograms (ECG): tips and tricks," International Journal of Cardiovascular Imaging, vol. 28, no. 6, pp. 1465-1475, Aug. 2012.

[3] J. W Krug, G. Rose, D. Stucht, G. D Clifford, and J. Oster, "Filtering the magnetohydrodynamic effect from 12-lead ECG signals using independent component analysis," in Computin in Cardiology (CinC). IEEE, 9-12 Sept. 2012, pp. 589-592.

[4] M. Zhang, M. Haritopoulos, and A. K. Nandi, "Fetal ECG subspace estimation based on cyclostationarity," in Signal Processing Conference (EUSIPCO), 2016 24th European. IEEE, 29 Aug. - 2 Sept. 2016, pp. 2060-2064.

[5] H. L. Hurd, "An investigation of periodically correlated stochastic processes," Ph.D. dissertation, Duke University, Nov. 1970.

[6] W. A. Gardner and L. Franks, "Characterization of cyclostationary random signal processes," IEEE Transactions on Information Theory, vol. 21 , pp. 4-14, 1975.

[7] J. Antoni, "Cyclostationarity by examples," Mechanical Systems and Signal Processing, vol. 23, pp. 987-1036, 2009.

[8] L. Lenart and M. Pipień, "Seasonality revisited - statistical testing for almost periodically correlated stochastic processes," Central European Journal of Economic Modelling and Econometrics, vol. 5, pp. 85-102, 2013.

[9] P. Demorest, "Cyclic spectral analysis of radio pulsars," of the Royal Astronomical Society, Monthly Notices, vol. 416, pp. 2821-2826, 2011.

[10] W. A. Gardner, A. Napolitano, and L. Paura, "Cyclostationarity: Half a century of research," Signal Processing, vol. 86, pp. 639-697, 2006.

[11] A. Napolitano, "Cyclostationarity: Limits and generalizations," Signal Processing, vol. 120, pp. 323-347, 2016.

[12] - "Cyclostationarity: New trends and applications," Signal Processing, vol. 120, pp. 385-408, 2016.

[13] R. Boustany and J. Antoni, "Cyclic spectral analysis from the averaged cyclic periodogram," in Proceedings of the 16th IFAC World Congress Prague Czech Republic, 2005. [Online]. Available: http://www.nt.ntnu. no/users/skoge/prost/proceedings/ifac2005/Fullpapers/03802.pdf

[14] J. W Krug, G. Rose, G. D Clifford, and J. Oster, "ECG-based gating in ultra high field cardiovascular magnetic resonance using an independent component analysis approach," Journal of Cardiovascular Magnetic Resonance, vol. 15, no. 104, Nov. 2013.

[15] C. Jutten and J. Herault, "Blind Separation of Sources, part i: An Adaptive Algorithm Based on Neuromimetic Architecture," Signal Processing, vol. 24(1), pp. 1-10, 1991.

[16] _ - "Blind Separation of Sources, part ii: Problems Statement," Signal Processing, vol. 24(1), pp. 11-20, 1991.

[17] P. Comon, "Independent component analysis, a new concept?" Signal Processing, Special Issue on Higher Order Statistics, vol. 36, no. 3, pp. 287-314, 1994.

[18] C. Capdessus and A. K. Nandi, "Extraction of a cyclostationary source using a new cost funcstion without pre-whitening," Signal Processing, vol. 91, no. 11, pp. 2497-2505, 2011.

[19] P. Comon and C. Jutten, Handbook of Blind Source Separation: Independent Component Analysis and Applications, Elsevier, Ed. Academic Press, 2010

[20] J. R. Keltner, M. S. Roos, P. R. Brakeman, and T. F. Bundiger, "Magnetohydrodynamics of blood flow," Magnetic Resonance in Medicine, vol. 16, pp. 139-149, October 1990. 